\title{
Sieving Effect of Agarose on Quasi-2D Silver Pattern Electroformation: A Pinning-Depinning-like Transition Resulting from Gelled Silver Plating Solutions
}

\author{
M. A. Pasquale, S. L. Marchiano, and A. J. Arvia* \\ Instituto de Investigaciones Físicoquimicas Teóricas y Aplicadas (INIFTA), Universidad Nacional de La \\ Plata-Consejo Nacional de Investigaciones Científicas y Técnicas, Sucursal 4, Casilla de Correo 16, \\ (1900) La Plata, Argentina
}

Received: December 12, 2003; In Final Form: April 12, 2004

\begin{abstract}
New features of silver patterns grown in quasi-2D electrochemical cells of parallel plate cathode/anode design utilizing sol and gel aqueous silver plating solutions are reported. Morphology transitions in the silver growth patterns that depend on the composition of plating solutions are observed. These transitions are explained by both a sieving effect due to the presence of agarose and the change in the relative contribution of diffusion and advection to the mass-transport-controlled electrochemical process. Characteristic scaling lengths from growth patterns are related to both the gel structure and the geometry of electrodeposits. Gels consist of a percolated network of gel and randomly distributed colloidal particles, their size and velocity being represented by hyperbolic distribution functions. For silver plating gels a pinning-depinning transition in growth patterns is also observed. From the dynamic scaling analysis of growth pattern 2D profiles, the critical growth and roughness exponent as well as the characteristic lengths of the environment were evaluated. Values of these exponents approach those predicted either by the Kardar, Parisi, and Zhang (KPZ) deterministic equation, or by the cellular automata lattice model that has been proposed for the dynamics of a driven interface in a medium with random pinning forces.
\end{abstract}

\section{Introduction}

The growth mode of solid phases is influenced by the characteristics of the substrate, the environment, and the operation routine. Accordingly, new solid phases with specific bulk and surface properties, such as crystallographic structure and roughness, and particle shape and size distribution for dispersed materials can be obtained by adjusting the working variables. These problems are of utmost importance in fastdeveloping areas of applied science related to materials science and engineering, for instance, in the conformal electrodeposition of metals ${ }^{1}$ and the design of new materials with preestablished properties. $^{2}$ To have a reliable fundamental support to these types of processes a key point is to understand the kinetics and mechanism of solid phase formation under a variety of environmental conditions.

To study the kinetics of a solid-phase growth such as the electrocrystallization of metals, a useful approach consists of following the growth pattern utilizing quasi-2D cells. ${ }^{3}$ Data from this type of experiments permit to distinguish changes in the kinetics of solid-phase growth at different length and time scales. ${ }^{4,5}$ The growth rate of the growing front depends on the electrochemical perturbation routine, the composition of the environment and its rheological properties, and the presence of impurities. The growth mode and transitions in the morphology of electrodeposits are also influenced by the dominant mass transport mechanism. ${ }^{6-8}$

Additives in metal plating solutions play a key role in the characteristics of the electrodeposits. They are involved in different physical and chemical mechanisms, such as additive adsorption at the growing front, and the formation of complex

* Corresponding author. E-mail address: ajarvia@inifta.unlp.edu.ar ion intermediates in the electrodeposition pathway. Additionally, the presence of colloidal additives in the plating solution introduces some kind of obstacles for the growing front displacement, producing a sort of sieving effect that may influence the growth mode of the solid phase.

In contrast to abundant data available for metal electrodeposition utilizing conventional aqueous plating baths, data for the electrochemical growth of solids in colloidal systems are very scarce. In fact, data is available only for copper electrodeposition from carboxi-methyl-cellulose-containing aqueous plating baths employing either three-dimensional, $3 \mathrm{D},{ }^{9}$ or quasi-2D electrochemical cells. ${ }^{10}$ In these cases a remarkable influence from the colloidal environment on the growth mode of metal electrodeposits was observed.

On the other hand, for non-Newtonian fluids, such as agarosecontaining media, the apparent viscosity of sols increases with agarose concentration to reach a value in which gels are produced. The high apparent viscosity of gels is able to overcome buoyancy forces acting at the metal growth front. ${ }^{11-13}$ Under these conditions one would expect that the effect of sieving on the growth front will be comparable to that of a random pinning force quenching to some extent the thermal noise of depositing metal ions. Accordingly, pinning forces offer a resistance against the growth front displacement.

In this work, novel features of silver growth patterns produced by quasi-2D electrochemical cells of parallel-plate cathodeanode design, utilizing different plating baths under the form of agarose sols and gels, are reported.

Silver branched patterns produced in supporting electrolytecontaining plating baths show a decrease in the number of branches, accompanied by an increase in their average thickness. These changes in the pattern morphology can be explained by the complex sieving effect due to the presence of agarose that 


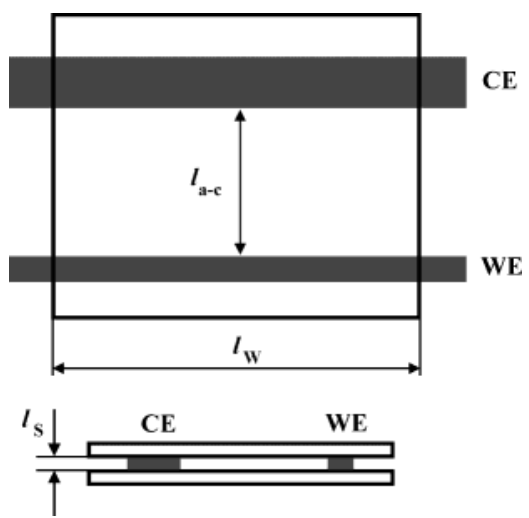

Figure 1. Top and lateral view of the quasi-2D and rectangular electrochemical cells. $\mathrm{WE}=$ working electrode; $\mathrm{CE}=$ counter electrode; $l_{\mathrm{s}}=$ thickness of the solution layer.

modifies the relative contribution of diffusion, advection, and/ or free convection in the mass-transport-controlled process. The characteristic scale lengths of silver patterns are related to the structural parameters of gels. The structure of agarose gels can be described as a percolated network of colloidal material with randomly distributed colloidal agglomerates, their size distribution following a hyperbolic function and their velocity decreasing with the agglomerate size. ${ }^{14-17}$

A scheme of the growing front is advanced. It consists of an immobile percolated network, and small mobile agglomerates, although their mobility is smaller than that of ionic species in the "agarose-free" domains of the plating bath. When the driven force for silver electrodeposits overcomes pinning, the abrupt change in the velocity of the growing front is consistent with a pinning-depinning-type transition.

The dynamic scaling analysis of 2D profiles from growth patterns allowed us to evaluate the growth and roughness exponents, as well as the characteristic lengths of the environment. Values of these exponents approach the prediction of the Kardar, Parisi, and Zhang (KPZ) deterministic equation including a random noise term as well as that of a cellular automata lattice model that has been proposed for the dynamics of a driven interface in a medium with random pinning forces. ${ }^{18,19}$ To clear this ambiguity further work is in progress.

\section{Experimental Section}

Quasi-2D rectangular cells with a vertical plate electrode arrangement were utilized to follow the evolution of growth patterns resulting from silver electrodeposition. Each rectangular cell consisted of top and bottom parallel flat plates made of either Lucite or glass separated by the distance $l_{\mathrm{s}}=0.025 \mathrm{~cm}$ and a parallel-edge silver (99.9\% purity) cathode-anode arrangement (width $l_{\mathrm{w}}=5 \mathrm{~cm}$, cathode-to-anode separation distance $l_{\mathrm{c}-\mathrm{a}}=2 \mathrm{~cm}$; Figure 1).

Silver growth patterns were obtained from $x \mathrm{M}$ silver sulfate $(0.008 \leq x \leq 0.024)+c_{\text {aga }} \% \mathrm{w} / \mathrm{v}$ agarose $\left(0 \leq c_{\text {aga }} \leq 0.6\right)$ aqueous solution, with addition of $0.5 \mathrm{M}$ sodium sulfate +0.01 M sulfuric acid as supporting electrolyte. Plating solutions, either as sols or gels, were prepared at the appropriate range of $c_{\text {aga, }}$, considering that the critical micellar concentration of agarose $(\mathrm{MW} \approx 120,000)$ is $c_{\mathrm{aga}}{ }^{*}=0.12 \% \mathrm{w} / \mathrm{v} .{ }^{12}$ Analytical reagent grade chemicals and Milli-Q water were used for plating solution preparation. These solutions were used after nitrogen saturation.

Agarose-containing solutions (sol, $c_{\mathrm{aga}}<c_{\mathrm{aga}}{ }^{*}$ ) were made by first dissolving silver sulfate and sodium sulfate in water under heating followed by agarose addition under stirring. As the system was cooling, drops of concentrated sulfuric acid were added slowly. For gels $\left(c_{\mathrm{aga}}>c_{\mathrm{aga}}{ }^{*}\right)$ two procedures were followed. For the in situ procedure (hereafter denoted as "gel in situ"), as the plating solution attained about $50{ }^{\circ} \mathrm{C}$, it was poured into the cell and allowed to reach the gel-forming temperature there. For the ex situ procedure (hereafter called "gel ex situ"), gel formation was made outside the cell, and the electrochemical cell was filled up by pasting and pressing the gel between its plates to ensure good electrical contacts at each electrode/environment interface.

Cathodic polarization curves at $0.30 \mathrm{~V} \mathrm{~s}^{-1}$, utilizing different plating solutions, allowed us to establish the potential range where the rate of the electrochemical reaction was under masstransport control. Polarization curves were plotted as cathodic current $\left(I_{\mathrm{c}}\right)$ versus cathode-to-anode voltage $\left(\Delta E_{\mathrm{c}-\mathrm{a}}\right)$. Values of $\Delta E_{\mathrm{c}-\mathrm{a}}$ are referred to the anode since in our case the anodic overvoltage for silver electrodissolution is negligible. The cathodic overpotential $\left(\eta_{\mathrm{c}}\right)$ was corrected for the ohmic drop, i.e., $\eta_{\mathrm{c}}=\Delta E_{\mathrm{c}-\mathrm{a}}-I_{\mathrm{c}} R_{\mathrm{c}}, R_{\mathrm{c}}$ being the ohmic resistance between the cathode and the anode. Because of the high value of the exchange current density of the $\mathrm{Ag}^{+}(\mathrm{aq}) / \mathrm{Ag}$ electrode, ${ }^{20}$ for $\eta_{\mathrm{c}} \leq-0.20 \mathrm{~V}, \eta_{\mathrm{c}}$ becomes practically equal to the concentration overpotential of the silver electrode.

Silver growth patterns were produced at constant $\Delta E_{\mathrm{c}-\mathrm{a}}$ in the range $-1.20 \leq \Delta E_{\mathrm{c}-\mathrm{a}} \leq-0.38 \mathrm{~V}$, for different electrodeposition times $(t)$. Simultaneously, the cathodic current $\left(I_{\mathrm{c}}\right)$ and charge $\left(Q_{\mathrm{c}}\right)$ transients were recorded using a Radiometer 32 potentiostat. Runs were performed at $298 \mathrm{~K}$.

Silver pattern morphology, root-mean-square roughness $\left(W_{\mathrm{rms}}\right)$, and velocity of the growing front were determined from the profile of pictures obtained with a stereoscopic microscope (Stemi 200 Zeiss) coupled to a camera (CCD Hitachi 220), connected to a video screen and a computer equipped with a frame grabber and an image analyzer (Contron Electronics KS300). Occasionally, a sequence of photographs was taken using a Contax camera 167 MT coupled to the microscope.

To estimate the size distribution of the colloidal agglomerates, Rhodamin B-stained gel samples were observed by confocal laser scanning microscopy (CLSM) using an Olympus FV 300 microscope.

\section{Results}

3.1. Characteristics of Agarose-Containing Sols and Gels. From the rheological standpoint, agarose-containing aqueous $0.01 \mathrm{M}$ sulfuric acid is sufficiently stable to be used for a few hours without a noticeable decrease in the flow time $\left(t_{\mathrm{f}}\right)$ determined with a viscometer. ${ }^{11,13}$ Agarose sols, either freshly prepared or after aging for a certain time, behave as nonnewtonian fluids, whereas gel-like structures obey Bingham plastic flow. ${ }^{12}$ It should be noted that for $c_{\text {aga }} \geq 0.02 \% \mathrm{w} / \mathrm{v}$, the increase in $t_{\mathrm{f}}$ can be related to a significant increase in the percent of gel structure. It has been shown that the relative percent of free agglomerates $\left(X_{\mathrm{L}}\right)$ depends on $c_{\text {aga }}$ according to equation $^{12}$

$$
X_{\mathrm{L}}=1-0.21 c_{\mathrm{aga}}^{1 / 2}
$$

which indicates the occurrence of at least two distinguishable structural domains in both sols and gels. Thus, for $c_{\text {aga }}=0.05 \%$ $\mathrm{w} / \mathrm{v}(\mathrm{sol})$, eq 1 yields $X_{\mathrm{L}}=0.95$, and for $c_{\mathrm{aga}}=0.6 \% \mathrm{w} / \mathrm{v}(\mathrm{gel})$, $X_{\mathrm{L}}=0.85$.

Small magnification pictures from "gel in situ" and "gel ex situ" (Figure 2) show a structure dominated by agglomerates and islands greater than $2000 \mu \mathrm{m}^{2}$, the largest islands being observed for "gels in situ". Conversely, due to the mechanical 

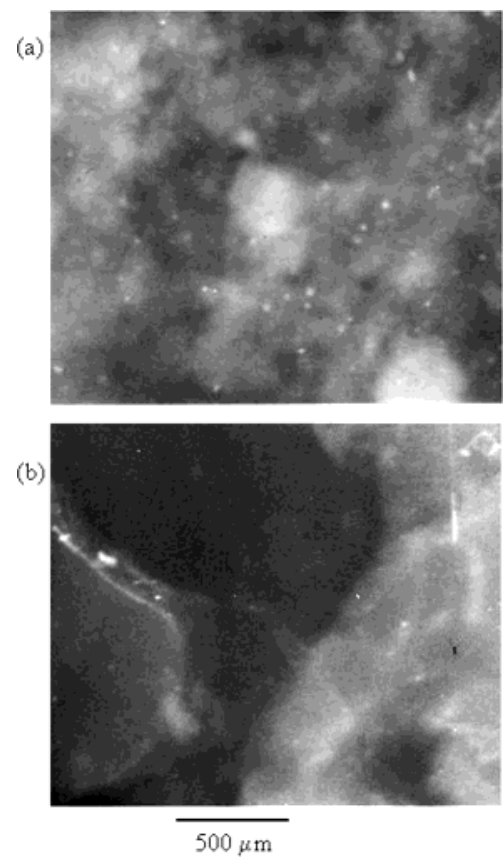

Figure 2. Photographs of $0.024 \mathrm{M}$ silver sulfate $+0.5 \mathrm{M}$ sodium sulfate $+0.01 \mathrm{M}$ sulfuric acid $+0.6 \% \mathrm{w} / \mathrm{v}$ agarose gel layers from ex situ (a) and in situ (b) procedures.

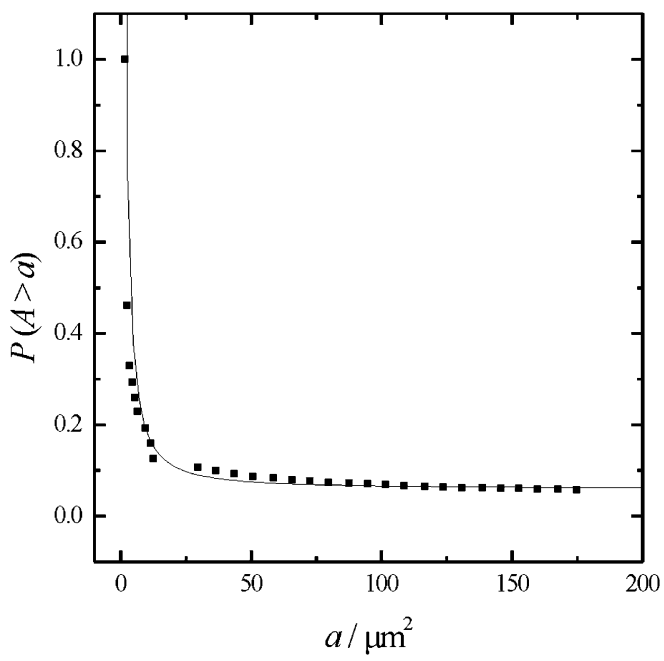

Figure 3. Plot of $P(A>a)$, the probability of appearance of agglomerates of area $A$ greater than the minimum area $a$, versus $a$. (ם) Experimental data. The curve represents the Korcak empirical law.

manipulation involved in the preparation method, "gels ex situ" show an agglomerate size distribution wider than that of "gels in situ".

Images of both gels (Figure 2a,b) confirm the appearance of randomly distributed percolated islands forming channels that are occupied by a large number of randomly distributed small agglomerates. Therefore, agarose gels can be described as a percolated, almost immobile structure with a large number of small agglomerates.

Data from both conventional images and CLSM photographs were plotted as a size distribution function of agglomerates with area $(A)$ larger than a value $(a)$ versus $a$ (Figure 3 ). According to the Korcak empirical law, ${ }^{21}$ the probability $P(A>a)$ of an agglomerate area $A$ exceeding a minimum area $a$ is given by the hyperbolic function

$$
P(A>a)=C a^{-B}
$$

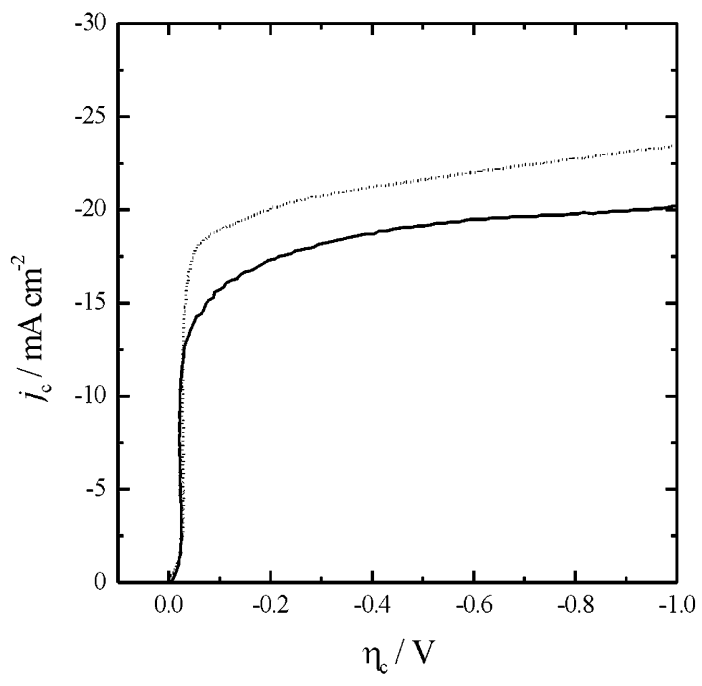

Figure 4. Cathodic polarization curves $\left(0.03 \mathrm{mV} \mathrm{s}^{-1}\right)$ after ohmic drop correction; $0.024 \mathrm{M}$ silver sulfate $+0.5 \mathrm{M}$ sodium sulfate $+0.01 \mathrm{M}$ sulfuric acid (dotted trace); $0.024 \mathrm{M}$ silver sulfate $+0.5 \mathrm{M}$ sodium sulfate $+0.01 \mathrm{M}$ sulfuric acid $+0.6 \% \mathrm{w} / \mathrm{v}$ agarose gel (solid trace). Quasi-2D rectangular cell; $298 \mathrm{~K}$.

In our case, the Korcak empirical law is fulfilled for $C=2.5$ and $B=1.3$. A similar decaying distribution function has been observed for gels formed from other carageen derivatives. ${ }^{22}$ From eq 2, as the fractal dimension is $D_{\mathrm{F}}=2 B$, it results in $D_{\mathrm{F}}$ $\cong 2.5$, as expected for a percolation process under chemical bonding control. ${ }^{21}$

For $c_{\text {aga }}>0.2 \% \mathrm{w} / \mathrm{v}$, the agglomerate size distribution (Figure 3 ) starts from an agglomerate average diameter of about $1 \mu \mathrm{m}$, the minimum size that can be discriminated and associated with agarose agglomerates from CLSM photographs. Accordingly, assuming a spherical agglomerate of agarose $(\mathrm{MW}=120,000)$ of radius $0.5 \times 10^{-4} \mathrm{~cm}$, the average volume of each agglomerate is $5.2 \times 10^{-13} \mathrm{~cm}^{3}$. Further, considering that the apparent gel density is close to 1 and the average weight of a single agarose molecule is $2 \times 10^{-19} \mathrm{~g}$, the average numbers of agarose and water molecules in $1 \mu \mathrm{m}$ diameter agglomerates are $2.6 \times 10^{6}$ and $1.0 \times 10^{9}$, respectively. The size range of these agglomerates agrees fairly well with those that have been reported elsewhere. ${ }^{15,22}$

3.2. Electrochemical Data. Cathodic polarization curves from either agarose-free or agarose-containing plating solution with an excess of supporting electrolyte show well-defined cathodic limiting currents $\left(I_{\mathrm{c}, \text { lim }}\right.$; Figure 4$)$. For the agarose-free solution $I_{\mathrm{c}, \lim }=22.7 \mathrm{~mA} \mathrm{~cm}^{-2}$, and for the agarose-containing bath $I_{\mathrm{c}, \lim }$ $=19.7 \mathrm{~mA} \mathrm{~cm}^{-2}$. This difference in $I_{\mathrm{c}, \lim }$ is consistent with a change in the mechanism of silver electrodeposition caused by the suppression of macroscopic flux in gelled media and by a slight change in the solvodynamic radius of ionic species in solution that may result from hydrogen bonding interactions with agarose molecules at the hydration sheath of ions.

Data from potentiostatic current transients for gels with different $c_{\mathrm{Ag}_{2} \mathrm{SO}_{4}}$ were displayed as $Q_{\mathrm{c}} / I_{\mathrm{c}}$ versus $t$ plots (Figures 5 and 6). Despite the current fluctuations due to stochastic disorder in the colloidal environment, data from potentiostatic current transients for "gels in situ" (Figure 5) tend to approach a straight line with slope 1 for $t<100 \mathrm{~s}$. The same slope results from data of "gels ex situ" for $t<15 \mathrm{~s}$ (Figure 6). In the former case, the fastest displacement of the growing front in the direction perpendicular to the electrode surface is observed, as expected for the more open structure of the environment. Conversely, data resulting from "gels in situ" (Figure 5) for $t$ 

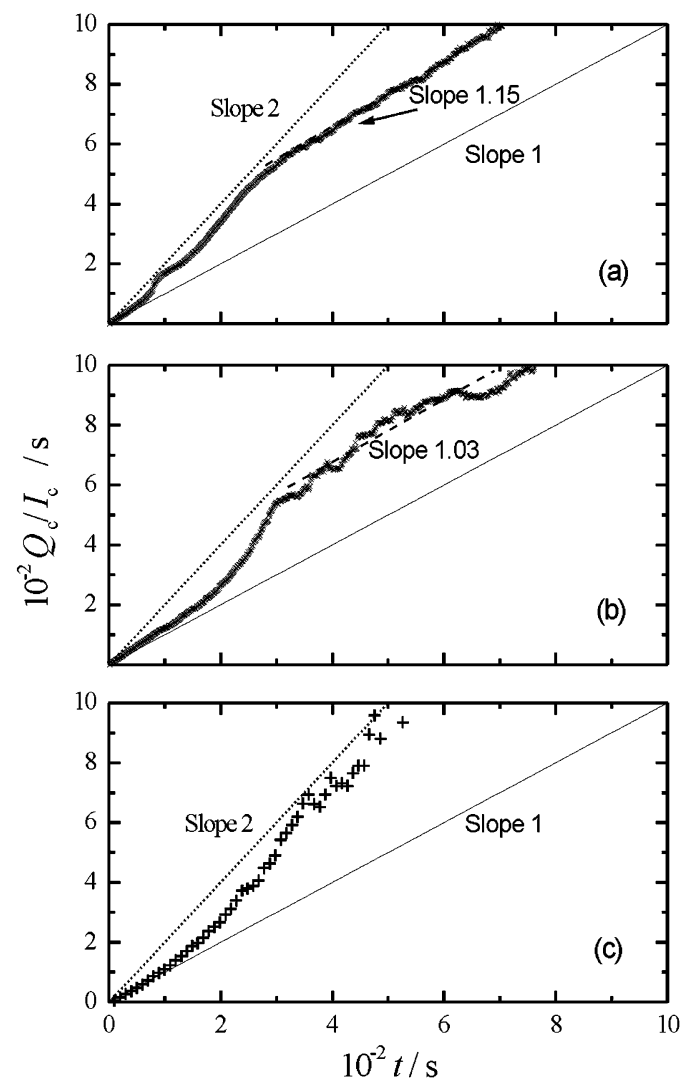

Figure 5. $Q_{\mathrm{c}} / I_{\mathrm{c}}$ versus $t$ plots obtained with the quasi-2D rectangular cell; $\Delta E_{\mathrm{c}-\mathrm{a}}=-1.2 \mathrm{~V}, x \mathrm{M}$ silver sulfate $+0.5 \mathrm{M}$ sodium sulfate + $0.01 \mathrm{M}$ sulfuric acid $+0.6 \% \mathrm{w} / \mathrm{v}$ agarose "gel in situ", (a) $x=0.016$; (b) $x=0.018$; (c) $x=0.024 ; 298 \mathrm{~K}$.

$>100 \mathrm{~s}$ tend to approach the straight line with slope 2, and a similar slope results from "gels ex situ" for $15 \leq t \leq 100 \mathrm{~s}$. For $t>100 \mathrm{~s}$, both gels approach again the slope 1 . This change in the slope of the $Q_{\mathrm{c}} / I_{\mathrm{c}}$ versus $t$ plots can be related to a change in the dominant mass-transport mechanism from diffusion to advection, influenced to some extent by the change in surface area of the cathode. ${ }^{23}$

3.3. Silver Pattern Morphology. Growth patterns produced at $\Delta E_{\mathrm{c}-\mathrm{a}}=-1.20 \mathrm{~V}$ from agarose-free $0.024 \mathrm{M}$ silver sulfate $+0.5 \mathrm{M}$ sodium sulfate $+0.01 \mathrm{M}$ sulfuric acid baths (Figure 7a) involve an initial first quasi-compact silver layer about 0.05 $\mathrm{mm}$ thick (not observable at the scale of the photograph) that appears for $0 \leq t \leq 15 \mathrm{~s}$. Subsequently, a second branched layer about $0.20 \mathrm{~mm}$ thick is formed for $15 \leq t \leq 30 \mathrm{~s}$. The electrodeposit morphology consists of protrusions that turn into branches growing in the direction of the electric field. For $t>$ $30 \mathrm{~s}$, the growing pattern consists of separated dense trees growing in the same preferred direction, partially screening the growth of smaller neighbor trees and columns.

On the other hand, growth patterns from $0.024 \mathrm{M}$ silver sulfate $+0.5 \mathrm{M}$ sodium sulfate $+0.01 \mathrm{M}$ sulfuric acid $+0.6 \%$ w/v agarose ("gel in situ") (Figure 7c) for $t<15 \mathrm{~s}$ consist of a first a quasi-compact silver layer, whereas for $t>15 \mathrm{~s}$, protrusions and branching are observed. In this case, the average interbranch distance is shorter than that observed for "gels ex situ". Additionally, for $45 \leq t \leq 1275 \mathrm{~s}$, a pinning effect on the growing front is evident. Finally, for $t>1300 \mathrm{~s}$, only a few branches continue growing, displaying a dense fan-like branched pattern.

Similar experiments run in "gel ex situ" at $\Delta E_{\mathrm{c}-\mathrm{a}}=-1.20$ $\mathrm{V}$ (Figure $7 \mathrm{~b}$ ) show, for $t<60 \mathrm{~s}$, the formation of a large number of protrusions merging from the initial quasi-compact
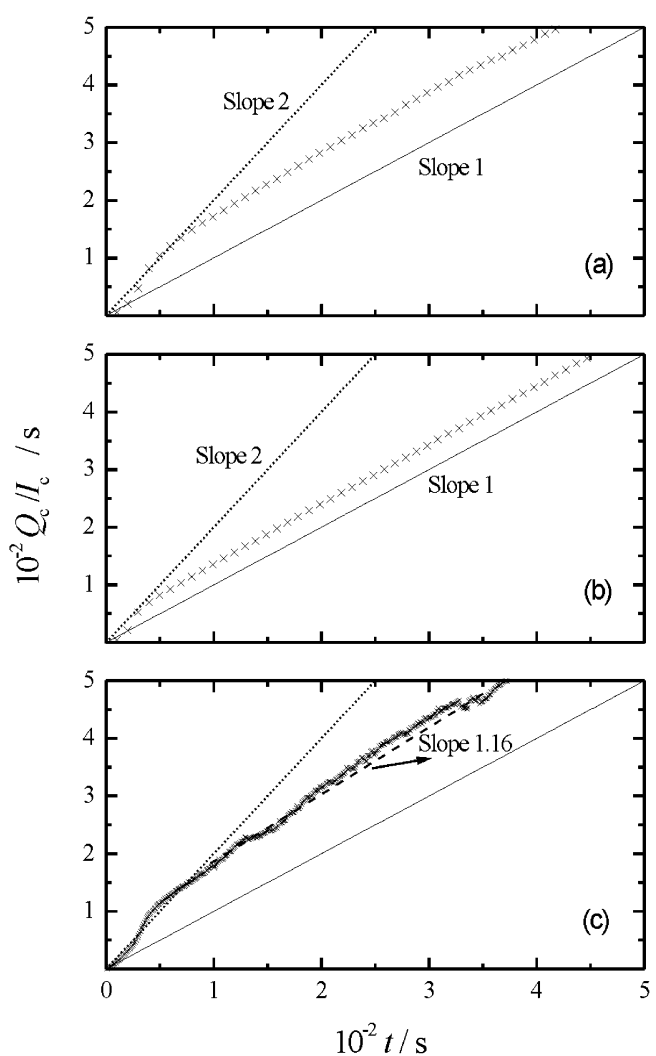

Figure 6. $Q_{\mathrm{c}} / I_{\mathrm{c}}$ versus $t$ plots obtained with the quasi-2D rectangular cell; $\Delta E_{\mathrm{c}-\mathrm{a}}=-1.2 \mathrm{~V}, x \mathrm{M}$ silver sulfate $+0.5 \mathrm{M}$ sodium sulfate + $0.01 \mathrm{M}$ sulfuric acid $+0.6 \% \mathrm{w} / \mathrm{v}$ agarose "gel ex situ". (a) $x=0.008$; (b) $x=0.012$; (c) $x=0.018 ; 298 \mathrm{~K}$.
( a )

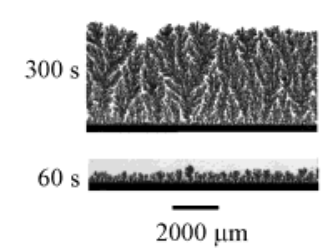

(c)
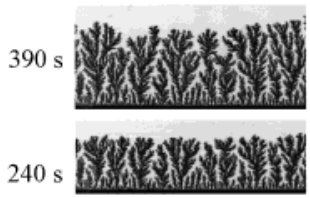

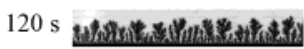

$0 \mathrm{~s}$

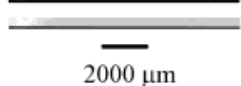

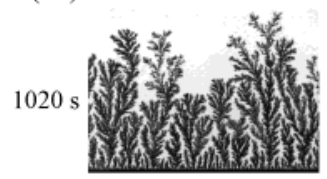

(b)

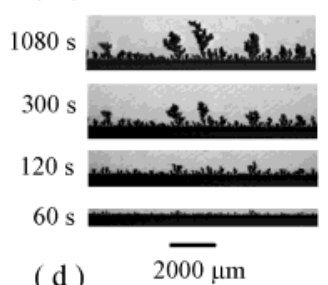

(d)
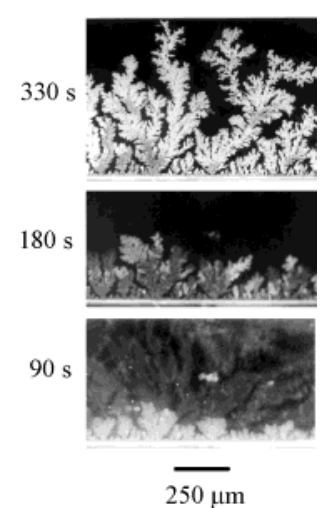

Figure 7. Sequences of silver patterns obtained in the quasi-2D rectangular cell: $\Delta E_{\mathrm{c}-\mathrm{a}}=-1.2 \mathrm{~V}$, (a) $0.024 \mathrm{M}$ silver sulfate $+0.5 \mathrm{M}$ sodium sulfate $+0.01 \mathrm{M}$ sulfuric acid; (b) idem $+0.6 \% \mathrm{w} / \mathrm{v}$ agarose "gel ex situ"; (c) idem $+0.6 \%$ w/v agarose "gel in situ". (d) Details of the growth sequence from (c). $298 \mathrm{~K}$. For $t=90 \mathrm{~s}$, channels can be clearly distinguished in (d). The influence of gel structure on the electrodeposit morphology can be appreciated.

silver layer. The number density of these protrusions decreases rapidly with $t$ due to coalescence of a number of neighboring protrusions and hindrance of further growth for others. Subse- 
(a)
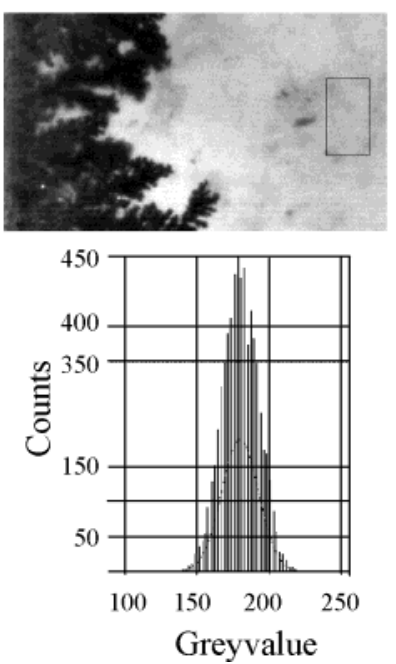

(b)
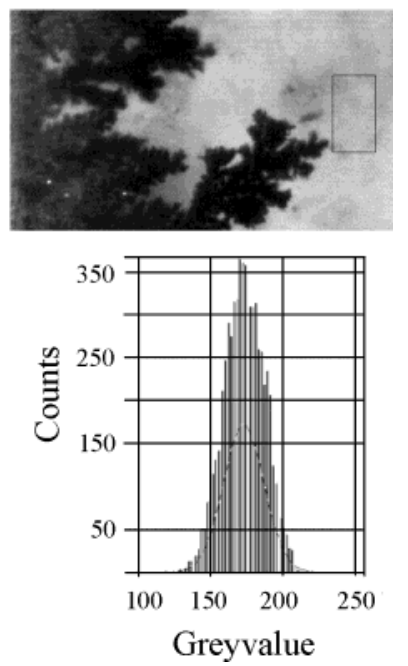

Figure 8. Images of silver quasi-2D growth patterns formed from 0.024 $\mathrm{M}$ silver sulfate +0.5 sodium sulfate $+0.01 \mathrm{M}$ sulfuric acid $+0.6 \%$ $\mathrm{w} / \mathrm{v}$ agarose gel at $\Delta E_{\mathrm{c}-\mathrm{a}}=-1.20 \mathrm{~V}$ and $298 \mathrm{~K}$, and their corresponding histograms. A reference box was drawn at the border of a spot of methylene blue-stained gel opposite to the cathode. (a) Grayscale histogram obtained either in the absence of a silver electrodeposit or when the latter was still far from the reference box edge. (b) Histogram obtained when the moving front was just ready to touch the reference box edge. In this case, the gray density (darker pixels) on the left-hand side of the reference box has notoriously increased. Note the significant change in both the half width and symmetry of the histogram, as well as the shift in its maximum value.

quently, for $t>200 \mathrm{~s}$, only a few columns dominate the growth pattern, and for $t>500 \mathrm{~s}$, the average velocity of the growing front perpendicular $(\langle V\rangle)$ to the initial electrode surface diminishes, yielding wider column tips.

The correlation between pattern morphology and gel structure (Figure 7d) confirms, in these cases, the key role of the presence of agarose-containing environment in determining the growth mode of silver electrodeposits (Figures 5 and 6). The average electrodeposition rate slows down considerably at channels and voids with a high density of small agglomerates, in contrast to free channels where fast branching is accomplished. The pinning effect is reflected throughout the change in the apparent density of silver electrodeposits, particularly in gelled plating solutions.

3.4. Mobility of Small Agarose Particles. The agarose agglomerate motion hypothesis in gels was demonstrated by running the following experiment in which the distribution of preset blue methylene-stained spots was disturbed by the growing front itself. For this purpose, "gel ex situ" containing methylene blue-stained spots was located approximately 0.25 $\mathrm{cm}$ away from the working electrode. The comparison of pictures before and after silver electrodeposition allowed us to detect changes in the distribution of stained spots caused by the motion of the growing front. We selected a $700 \times 700 \mu \mathrm{m}$ rectangular region of the image (open drawn down rectangle in Figure 8) so that one-half of the stained spots were allocated inside the selected region and the rest were placed between this region and the working electrode surface. A first grayscale histogram (blank) was constructed for pixels inside the box, providing information about how stained agglomerates (darker ones) were distributed in the selected region. Then, silver electrodeposition was started, and as the growing front reached spots just outside the selected region, another grayscale histogram was made. The latter showed the appearance of a larger
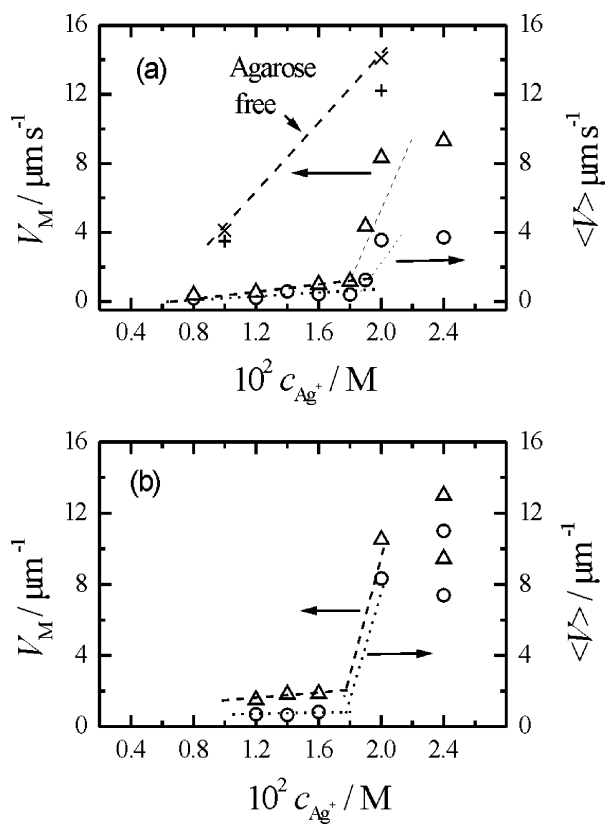

Figure 9. Plots of $\langle V\rangle$ and $V_{\mathrm{M}}$ versus $c_{\mathrm{Ag}_{2} \mathrm{SO}_{4}}$ from $x$ M silver sulfate $+0.5 \mathrm{M}$ sodium sulfate $+0.01 \mathrm{M}$ sulfuric acid at $\Delta E_{\mathrm{c}-\mathrm{a}}=-1.2 \mathrm{~V}$, $(+)$ agarose-free solution; $(\Delta) 0.6 \% \mathrm{w} / \mathrm{v}$ agarose "gel ex situ"; $(\mathrm{O})$ $0.6 \% \mathrm{w} / \mathrm{v}$ agarose "gel in situ"; $298 \mathrm{~K}$.

number of darker pixels in the reference box (Figure 8), as one should expect from the displacement of smaller agglomerates pushed ahead by the growing front. From these results, it is reasonable to consider that small nonpercolated moving agglomerates interfere with the displacement of the growing front, i.e., the local rate of silver electrodeposition.

3.5. Influence of Silver Ion Concentration on the Velocity of the Growing Front. In our experiments, the driven force of the electrochemical process is the concentration gradient of silver ions at the cathode/solution interface. Correspondingly, to investigate the influence of the driven force on the pattern morphology, runs setting $\Delta E_{\mathrm{c}-\mathrm{a}}=-1.2 \mathrm{~V}$ and $c_{\mathrm{aga}}$ in the range $0.008 \leq c_{\mathrm{Ag}_{2} \mathrm{SO}_{4}} \leq 0.024 \mathrm{M}$ (the solubility of silver sulfate at $298 \mathrm{~K})$ were made.

The plots of both the average velocity of the growing front perpendicular to the initial electrode surface $\langle V\rangle$, and the maximum growing front velocity in the same direction $V_{\mathrm{M}}$, versus $c_{\mathrm{Ag}_{2} \mathrm{SO}_{4}}$ for agarose-free solution (Figure 9a,b) show a linear increase in both $\langle V\rangle$ and $V_{\mathrm{M}}$ with $c_{\mathrm{Ag}_{2} \mathrm{SO}_{4}}$. Conversely, for both gels "in-situ" and "ex-situ" and $c_{\mathrm{Ag}_{2} \mathrm{SO}_{4}}<1.7 \times 10^{-2} \mathrm{M}$, the $V_{\mathrm{M}}$ versus $c_{\mathrm{Ag}_{2} \mathrm{SO}_{4}}$ and $\langle V\rangle$ versus $c_{\mathrm{Ag}_{2} \mathrm{SO}_{4}}$ plots show a slow increase in both $V_{\mathrm{M}}$ and $\langle V\rangle$ with $c_{\mathrm{Ag}_{2} \mathrm{SO}_{4}}$, whereas for $\left.c_{\mathrm{Ag}_{2} \mathrm{SO}_{4}}\right\rangle$

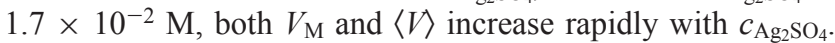
Correspondingly, there is a critical concentration of silver sulfate, $c^{*}{ }_{\mathrm{Ag}_{2} \mathrm{SO}_{4}} \approx 1.7 \times 10^{-2} \mathrm{M}$, that is associated with a dramatic increase in the velocity of the growing front that approaches the value for agarose-free plating solution. This sudden change in the displacement of the growing front correlates with the morphology transition of silver electrodeposits. This transition starts from the slow growth of branched columns initially formed on the first compact thin silver layer, to the fast growth of a fan-like morphology of only a few large branched columns (Figure 7). Then, the morphology transition that appears for $c_{\mathrm{Ag}_{2} \mathrm{SO}_{4}}>\mathrm{c}^{*} \mathrm{Ag}_{2} \mathrm{SO}_{4}$ indicates that the driving force of the electrochemical reaction becomes sufficiently large to overcome the resistance imposed by the sieving effect due to the presence of agarose. Hence, the overall effect resembles that of a pinning-depinning transition that has been observed in the growth of solid phase in porous media. ${ }^{24}$ 


\section{Discussion}

4.1. Fundamental Aspects of Agarose Sols and Gels. Electrochemically produced silver patterns depend on both the sol and gel structure and composition of the plating solution. For aqueous agarose sols, domains with different structures (Figure 2) can be explained via specific inter- and intramolecular interactions of $\mathrm{OH}$ groups from agarose molecules and water. Agarose is an alternating polysaccharide copolymer of alga origin, its backbone containing $(1 \rightarrow 4)$ and $(1 \rightarrow 3)$ linked 3,6anhydro- $\alpha$-L-galactose. ${ }^{25-27}$ The 4 -linked residues give rise to an extended ribbon, whereas the 3-linked units generate a double helix consisting of parallel 3-fold chains with a left-hand direction and a pitch of $1.90 \mathrm{~nm}$. A large part of the agar backbone can be replaced by neutral and charged groups. This structure can persist under hydrated conditions in sol and gel three-dimensional networks. The formation of gels involves the association of chains through double helices to develop randomly coiled agglomerates in a three-dimensional network structure. ${ }^{25,26}$ In agarose the repeating sequence structure is interrupted by the occurrence of other residues. ${ }^{28}$ Such interruptions can produce a remarkable modification in bulk properties by terminating intermolecular association through structurally and hysterically regular junction zones, leading to a $3 \mathrm{D}$ aggregate formation. The same effect is also produced by departure from the regular primary sequence. Then, the interchain association through double helical junction zones is terminated by galactose residues in the normal, unbridged ring form in place of the fused anhydride ring structure. This introduces a backbone kink that cannot be accommodated within the ordered structure. ${ }^{25,28}$ The formation of this molecular organization, in particular intermolecular networks, has been proved by rheological studies of agarose sols and gels. ${ }^{12}$

The sieving effect caused by the presence of agarose in the plating bath depends on the disordered structure of sols and gels (Figures 2 and 3), which in turn depends on the preparation procedure. Data from both in situ and ex situ gels indicate that the sieving effect is equivalent to a pinning effect on the average rate of displacement of the growing front. The fractal structure of gels is consistent with an anisotropic rate of displacement of the growing front imposed by the pinning effect.

The pinning effect can be related to characteristic lengths for the growing phase in the direction perpendicular and parallel to the electrode surface, respectively. These lengths are related to the size of aggregates and channels in the environment. Then, the value of $c^{*} \mathrm{Ag}_{2} \mathrm{SO}_{4}$ related to the pinning-depinning transition in the growth mode of silver electrodeposits should be related to the critical lengths derived from the gel structure.

The gel structure can be better described as randomly distributed large quasi-frozen percolated structures and small mobile agglomerates, as depicted in Figure 10. The ratio between the average rate of displacement of small agglomerates and the average silver electrodeposit front rate becomes a relevant parameter that determines the global morphology of the electrodeposits and the kinetics of the process. In our case, the electrodeposition rate depends on the local concentration gradient of silver ions and can be modified by properly adjusting the concentration of silver ions in the plating bath. Then, the environment-dependent kinetics of silver pattern growth can be understood in terms of a random size distribution of pinning agglomerates (random walkers) covering a wide range of mobility that increases with the reciprocal of the average agglomerate radius.

The presence of agarose also alters the mass transport properties in the environment by suppressing free convection

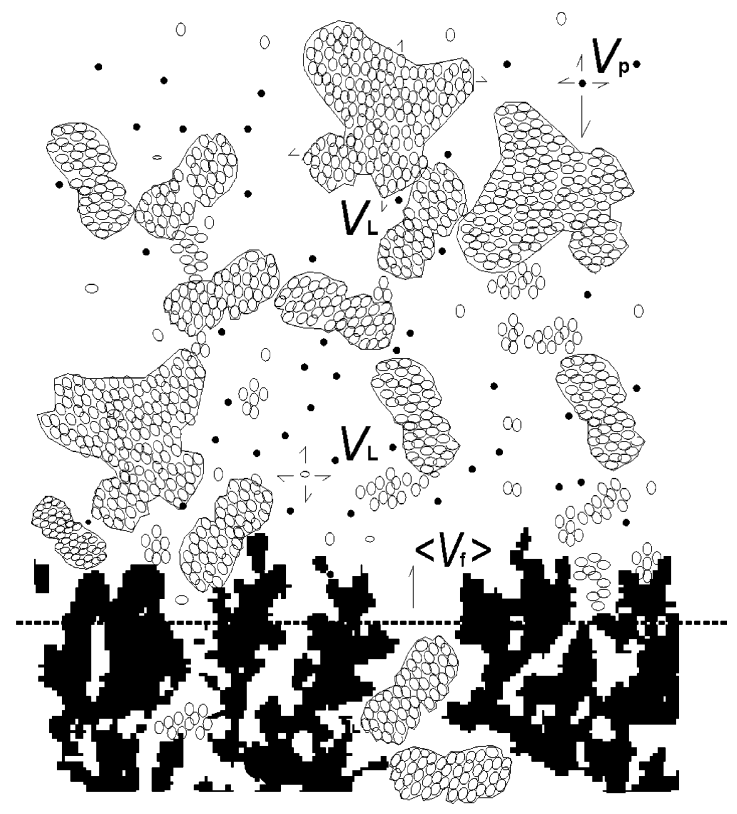

Figure 10. Scheme for silver electrodeposition in agarose gels. Colloidal particles are characterized by wide size and velocity distribution functions. $\langle V\rangle$ is the average moving front velocity; $V_{\mathrm{p}}$ is the velocity of electrodepositing cations; $V_{\mathrm{L}}$ denotes the velocity of agarose agglomerates. The dashed trace represents the average moving front line.

TABLE 1: Values of $K$ Estimated from the $Q_{\mathrm{c}} / I_{\mathrm{c}}$ versus $t_{\mathrm{c}}$ Plots for Gelled Silver Plating Solutions under Different Transport Mechanism on an Initially Planar Surface

\begin{tabular}{cll}
\hline mechanism & \multicolumn{1}{c}{ area } & $K$ \\
\hline pure diffusion & $\mathrm{S}(t)=$ constant & 2 \\
pure diffusion & $\mathrm{S}(t)=m t$ & $2 / 3$ \\
advection & $\mathrm{S}(t)=$ constant & 1 \\
& $V_{\mathrm{f}}=$ constant & \\
advection & $\mathrm{S}(t)=m t$ & 0.5 \\
& $V_{\mathrm{f}}=$ constant &
\end{tabular}

and enhancing advection in going from agarose-free to gelled plating solution as concluded from potentiostatic current transient data plotted as $Q_{\mathrm{c}} / I_{\mathrm{c}}$ versus $t$ plots (Figures 5 and 6). For each regime, the relationship $Q_{\mathrm{c}} / I_{\mathrm{c}}=K t$ can be obtained to evaluate the proportionality constant $K$. For mass-transportcontrolled electrochemical processes, $K$ becomes overpotentialindependent but changes with the dominant mass-transport mechanism and evolution of the cathode area. ${ }^{4}$ Thus, for solid metal electrodeposition the ratio $Q_{\mathrm{c}} / I_{\mathrm{c}}$ is obtained from the expression

$$
\frac{Q_{\mathrm{c}}}{I_{\mathrm{c}}}=\frac{\int j_{\mathrm{c}} S \mathrm{~d} t}{j_{\mathrm{c}} S}
$$

In eq $3, S$ is the effective area of the cathode surface, $j_{\mathrm{c}}=I_{\mathrm{c}} / S$; $j_{\mathrm{c}}=j_{\mathrm{c}}(t)$ and $S=S(t)$. The expression $j_{\mathrm{c}}=j_{\mathrm{c}}(t)$ depends on whether the data correspond to the range of time in which diffusion, stationary free convection, or advection dominates mass transport, and $S(t)$ accounts for the evolution of $S$ during silver electrodeposition.

The stationary cathodic limiting current values of $K$ resulting from the preceding situations are assembled in Table 1. Their comparison to experimental data (Figures 5 and 6) provided an idea of the dominant mass-transport mechanisms during silver electrodeposition.

The dynamic scaling theory was applied to obtain relevant information about the characteristics of roughening, the domi- 
nant molecular mechanism determining silver pattern shape, and the occurrence of possible transitions in the growth mode.

4.2. Dynamic Scaling Analysis. The dynamic scaling theory predicts that the interface width of a growing front (profile), i.e., the mean square surface height fluctuation, $W(L, t)$ is defined by

$$
W_{\mathrm{rms}}=W(L, t)=\sqrt{\frac{1}{L_{\mathrm{i}=1}^{L}}[h(i, t)-\bar{h}(t)]^{2}}
$$

with $h(i, t)$ being the height of the $i$ column, and $\bar{h}(i, t)$ is the mean height

$$
\bar{h}(t)=\frac{1}{L} \sum_{i=1}^{L} h(i, t)
$$

For length scale $L$ and growing time $t, W(L, t)$ scales as $24,29,30$

$$
W(L, t) \propto L^{\alpha} f\left(t / L^{\alpha / \beta}\right)
$$

Equation 6 for $t \gg L^{\alpha / \beta}$ becomes

$$
W(L, t) \propto L^{\alpha}
$$

and for $t \ll L^{\alpha / \beta}$

$$
W(L, t) \propto t^{\beta}
$$

In equations $6-8, \alpha$ and $\beta$ are the roughness and growth exponent, respectively. The value of $\alpha$ is related to the surface texture of the deposit, and correspondingly, to the fractal surface dimension $D_{\mathrm{F}}$ of the self-affine surface by $D_{\mathrm{F}}=3-\alpha$. Thus, for $\alpha \rightarrow 1, D_{\mathrm{F}} \rightarrow 2$, the surface tends to be Euclidean (ordered), whereas when $\alpha \rightarrow 0, D_{\mathrm{F}} \rightarrow 3$, the surface exhibits an increasing degree of disorder. As exponents $\alpha$ and $\beta$ are interdependent, there is a simple way to collapse the temporal and spatial $W(L, t)$ data onto a single curve by plotting $W / L^{\alpha}$ versus $t / L^{\alpha / \beta}$.

Let us first consider silver growth patterns in supporting electrolyte-containing "gel in situ" $\left(c_{\text {aga }}=0.6 \% \mathrm{w} / \mathrm{v}\right)$. The $\log W(L, t)$ versus $\log t$ plot (Figure 11a) shows three ranges of $t$ where linear functionalities are fulfilled. For the range 1.15 $\leq \log t \leq 1.8$, the slope of the best linear function is $\beta=0.88$ \pm 0.15 ; for $1.8<\log t \leq 2.2, \beta=0.33 \pm 0.15$, and for $\log t$ $>2.2, \beta=0.88 \pm 0.2$. This plot shows two crossovers for log $t^{*}=1.85\left(t^{*}=70.8 \mathrm{~s}\right)$, and for $\log t^{*}=2.25\left(t^{*} \approx 180 \mathrm{~s}\right)$. On the other hand, data for $\log t>2.2$ were plotted as $\log W$ versus $\log L$ (Figure 11a) to evaluate $\alpha$. This plot exhibits two linear ranges of $\log L$ with different slopes, namely, for the range 2.25 $\leq \log L \leq 3.0, \alpha=1.25 \pm 0.15$; for $\log L>3.13$, the value of $W$ remains almost constant within $0.1 \log$ unit, i.e., $\alpha=0 \pm$ 0.1 . This plot defines a crossover for $\log L^{*} \approx 2.8\left(L^{*} \approx 790\right.$ $\mu \mathrm{m})$.

Similar plots from data obtained with the same "gel ex situ" $\left(c_{\mathrm{aga}}=0.6 \% \mathrm{w} / \mathrm{v}\right)$ led to a $\log W$ versus $\log t$ plot (Figure $11 \mathrm{~b}$ ) with two linear portions with different slopes, namely, $\beta=1$ \pm 0.2 for $1.9 \leq \log t \leq 2.5$ and $\beta=0.33 \pm 0.05$ for $\log t>$ 2.5 , and a crossover for $\log t^{*}=2.5\left(t^{*}=316 \mathrm{~s}\right)$. Otherwise, the $\log W$ versus $\log L$ plot (Figure 11b) also exhibits two linear portions, a first one for $\log L<2.95$ approaching $\alpha=1.25 \pm$ 0.05 , and a second one for $\log L>2.95$ with $\alpha=0 \pm 0.1$, and a crossover at $\log L^{*}=3\left(L^{*}=1000 \mu \mathrm{m}\right)$.

4.3. Scaling Analysis Data and Proposed Models. The dynamic scaling analysis of growth patterns raises the possibility of advancing in the interpretation of the main mechanism associated with silver electrodeposition in an environment with (a)
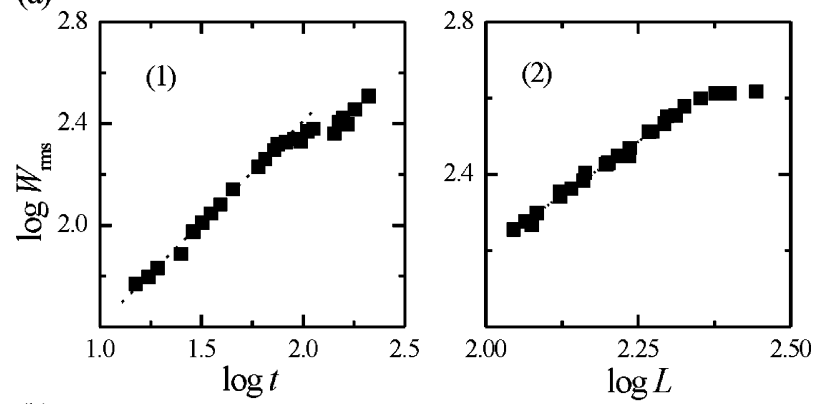

(b)
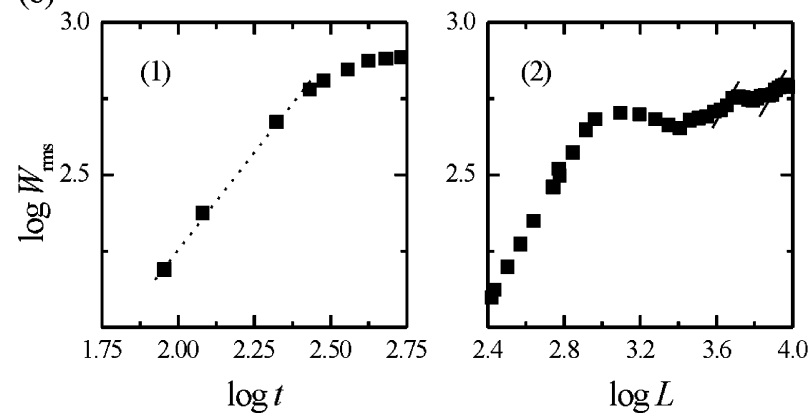

Figure 11. $\log W_{\text {rms }}$ versus $\log t$ and $\log W_{\text {rms }}$ versus $\log L$ plots from silver electrodeposit profiles: $\Delta E_{\mathrm{c}-\mathrm{a}}=-1.20 \mathrm{~V}$; (a) $0.024 \mathrm{M}$ silver sulfate $+0.5 \mathrm{M}$ sodium sulfate $+0.01 \mathrm{M}$ sulfuric acid $+0.6 \% \mathrm{w} / \mathrm{v}$ agarose "gel ex situ"; (b) idem $+0.6 \%$ w/v agarose "gel in situ", 298 K. Dashed traces represent the slopes referred to in the text.

randomly distributed quenched noise, and the size and velocity distribution of quenchers. For gels, irrespective of their preparation, the values $\alpha=1.25 \pm 0.2$ and $\beta=0.95 \pm 0.15$ are very different from $\alpha=0.63$ and $\beta=0.63$ resulting from either the simple directed percolation depinning (DPD) model or the DPD with some modifications. ${ }^{31-33}$

Despite data scattering and the limited extension of the linear relationship usually obtained from experimental data, scaling exponents measured for silver electrodeposition from both gels approach those predicted by either the Kardar, Parisi, and Zhang (KPZ) deterministic equation or by a cellular automata lattice model ${ }^{19,34}$ that has been proposed for the dynamics of a driven interface in a medium with random pinning forces. The scattering of data may be partly attributed to the irregular structure of gels. Additional data are, however, required to have the possibility to discriminate between these two models. The main drawback lies in the fact that the complexity of gel systems exceeds that of the models that have been proposed, particularly the occurrence of static and dynamic domains in the environment producing a combined pinning effect. This fact encourages further experimental work about these systems that will provide data to be used to advance in the system modeling.

\section{Conclusions}

By increasing agarose concentration in the plating solutions, the contribution of natural convection to the mass-transportcontrolled silver electrodeposition is progressively diminished. For gels, a pure diffusion control is reached, as could be concluded from the potentiostatic current transients and from $Q_{\mathrm{c}} / I_{\mathrm{c}}$ versus $t$ plots for a constant cathode area.

Gels consist of islands and channels that affect mass-transport properties of depositing ions and produce a sieving effect that is reflected in the electrodeposit growth pattern. Gels produce a randomly distributed quenched noise at the moving interface of the electrodeposit. A pinning-depinning transition appears 
by changing the driving force of the moving interface. There is a critical silver salt concentration associated with this transition.

Generally, silver-electroformed pattern morphology in a gelled environment is characterized by a relatively small number of wide branches with tip splitting, greatly influenced by the islandchannel structure of gels, which depends on the preparation method, i.e., ex-situ or in-situ. The complicated cathode area versus $t$ function is related to the gel structure.

From the dynamic scaling analysis of growth pattern 2D profiles, the critical growth and roughness exponents, as well as the characteristic lengths of the environment, were evaluated. These exponents approach the predictions of both the Kardar, Parisi, and Zhang (KPZ) deterministic equation, and a cellular automata lattice model that has been proposed for the dynamics of a driven interface in a medium with random pinning forces.

Results reported in this work are interesting in two main aspects. They provide, for the first time, quantitative information for the general problem of growth patterns of a solid phase under diffusion control. For this purpose, data derived from silver growing patterns, using silver electrodeposition as test process, offer the possibility of a first comparison to model data.

Acknowledgment. This work was financially supported by the Consejo Nacional de Investigaciones Científicas y Técnicas (CONICET) and PICT 98 No. 06-03251 from Agencia Nacional de Promoción Científica y Tecnológica of Argentina.

\section{References and Notes}

(1) Moffat, T. P.; Bonevich, J. E.; Huber, W. H.; Stanisheky, A.; Stafford, D. R.; Josell, D. J. Electrochem. Soc. 2000, 147, 4524.

(2) Fleury, V.; Barkey, D. Europhys. Lett. 1996, 36, 253.

(3) Matsushita, M.; Sano, M.; Hayakawa, Y.; Hongo, H.; Sawada, Y. Phys. Rev. Lett. 1984, 5, 286.

(4) . Pasquale, M. A.; Marchiano, S. L.; Arvia, A. J. J. Electroanal. Chem. 2002, 532, 255.

(5) . Pasquale, M. A.; Marchiano, S. L.; Arvia, A. J. J. Electrochim. Acta 2002, 48, 153.

(6) . Fleury, V.; Chazalviel, J.-N.; Rosso, M.; Sapoval, B. J. Electroanal. Chem. 1990, 290, 249.

(7) Kuhn, A.; Argoul, F. J. Electroanal. Chem. 1995, 397, 93.
(8) Barkey, D. P.; Watt, D.; Liu, Z.; Raber, S. J. Electrochem. Soc. 1994, 141, 1206

(9) Arvia, A. J.; Bazán, J. C.; Carrozza, J. S. W. Electrochim. Acta $1968,13,81$.

(10) Wang, M.; Ming Nai-ben; Bennema, P. Phys. Rev. E 1993, 48, 3825.

(11) Pasquale, M. A.; Marchiano, L. S.; Bolzán, A. E.; Arvia A. J. J. Appl. Electrochem. 2003, 33, 431.

(12) . Gavira Ruiz, J. M.; Novella, M. L.; Moreno, R.; Gavira, J. A. J. Cryst. Growth 2001, 232, 165.

(13) . Carro, P.; Marchiano, S. L.; Hernández Creus, A.; González, S.; Salvarezza, R. C.; Arvia, A. J. Phys. Rev. E 1993, 84, R2374.

(14) Starchev, K.; Sturm, J.; Weill, G.; Brogen, C.-H. J. Phys. Chem. B 1997, 101, 5659.

(15) Attwood, T. K.; Nelmes, B. J.; Sellen, D. B. Biopolymers 1988, $27,201$.

(16) Waki, S.; Harvey, J. D.; Bellamy, A. R. Biopolymers 1982, 21, 1909.

(17) Stellwagen, J.; Stellwagen, N. C. Biopolymers 1994, 34, 187.

(18) Leschhorn, H. Physica A 1993, 195, 324.

(19) Leschhorn, H., Ph.D. Thesis, Ruhr University, Bochum, 1994.

(20) Gerischer, H.; Tischer, R. P. Z. Electrochem. 1957, 61, 1159.

(21) Avnir, D. The Fractal Approach to Heterogeneous Chemistry, J. Wiley: New York, 1989; Ch. 5.

(22) Reid, D. S.; Bryce, T. A.; Clark, A. H.; Rees, D. A. Discuss. Faraday Soc. 1974, 57, 275.

(23) Pasquale, M. A.; Marchiano, S. L.; Vicente, J. L.; Arvia, A. J., (to be submitted).

(24) Barabási, A.-L.; Stanley, H. E. Fractal Concepts in Surface Growth, Cambridge University Press: New York, 1995.

(25) Rees, D. A.; Morris, E. D.; Thon, D.; Madden, J. K., In The Polysaccharides; Aspinall, G. O., Ed.; Academic Press: New York, 1982; Vol I, Ch. 5, p 195.

(26) Arnott, S.; Fulmer, A.; Scott, W. E. J. Mol. Biol. 1974, 90, 269.

(27) Rees, D. A.; Welsh, E. J. Angew. Chem., Int. Ed. Engl. 1977, 16, 214.

(28) Perlin, A. S.; Casu, B. In The Polysaccharides; Aspinall, G. O., Ed.; Academic Press: New York, 1982; Vol. I, Ch. 4, p 182.

(29) Godréche, C. Solids Far From Equilibrium; Cambridge University Press: Cambridge, England, 1990; Ch. 6.

(30) Family, F.; Vicsek, T. Dynamics of Fractal Surfaces; World Scientific: Singapore, 1991.

(31) Tang, L. H.; Leschorn, H. Phys. Rev. A 1992, 45, R8309.

(32) Buldyrev, S. V.; Barabási, A.-L.; Caserta, F.; Havlin, S.; Stanley,

H. E.; Vicsek, T. Phys. Rev. A 1992, 45, R8313.

(33) Leschhorn, H.; Tang, L. H. Phys. Rev. Lett. 1994, 49, 1238.

(34) Solé, R. V.; Manrubia, S. C., Orden y Caos en Sistemas Complejos, Universitat Politécnica de Catalunya, Spain, 1996. 